\title{
REDES NEURONALES ARTIFICIALES (RNAs) EN LA PREDICCIÓN DE PROPIEDADES TERMODINÁMICAS DEL LÍQUIDO Y VAPOR DE AGUA SATURADOS
}

\section{NEURAL NETWORKS (ANNS) IN PREDICTION OF THERMODYNAMIC PROPERTIES OF LIQUID AND SATURATED WATER VAPOR}

\author{
Fabián A. Ortega ${ }^{1}$, Mauricio J. Sierra² y Emiro A. Lopez ${ }^{2}$ \\ Recibido para publicación: 29 de mayo de 2012 - Aceptado para publicación: 23 de julio de 2012
}

\section{RESUMEN}

Las propiedades termodinámicas del líquido y vapor de agua saturados son útiles en el diseño de evaporadores, columnas de destilación, líneas de transporte de vapor e intercambiadores de calor en general, y se pueden modelar a través de ecuaciones tradicionales conocidas (Clapeyron, ClausiusClapeyron, Antoinne, Riedel y Watson, etc.), todas ellas arrojando una sola propiedad termodinámica, razón por la cual el modelo obtenido con RNAs es importante, ya que éste correlaciona muy bien todas las variables termodinámicas con la temperatura de saturación. El objetivo de este trabajo fue modelar las propiedades termodinámicas presión de saturación, entalpía, energía interna y entropía del líquido y vapor de agua saturados por medio de RNAs. Para el desarrollo de RNAs se utilizó la herramienta Simulink de Matlab 7.5, la arquitectura usada fue MLP (perceptrón multicapa), la función de transferencia usada en las neuronas ocultas fue la sigmoidea y en las de salida fue la función lineal, el algoritmo Levenberg-Marquardt backpropagation fue usado para el entrenamiento y proceso de aprendizaje de la red. El mejor ajuste de los datos $(R=0,999999, M S E=4,57)$ se obtuvo con la utilización de 7 neuronas ocultas en el modelo, el cual permite obtener las propiedades termodinámicas del líquido y vapor de agua saturados con sólo entrar la temperatura de saturación. Se encontró un modelo matemático de red neuronal que compite con los modelos termodinámicos tradicionales.

Palabras claves: red neuronal, vapor saturado, propiedades termodinámicas, modelo matemático.

\footnotetext{
${ }^{1}$ Ingeniero de Alimentos. Docente. Universidad de Córdoba. Montería, Colombia. ingenierofabianortega@gmail.com. Calle 11 Carrera 4 esquina Barrio San Pedro (Sahagún-Córdoba). Tel. 7775666. Tel. Cel. 300-5583559.

${ }^{2}$ Ingeniero de Alimentos. Docente. Universidad de Córdoba. Montería, Colombia.
} 
Fabián Ortega, Redes Neuronales Artificiales y predicción de propiedades

\section{ABSTRACT}

The thermodynamic properties of liquid and saturated water vapor are useful in design of evaporators, distillation columns, steam transport and heat exchange in general, and can be modeled by known traditional equations (Clapeyron, Clausius-Clapeyron, Antoinne, Riedel and Watson, etc.). Each of these yield a single thermodynamic property. This is why the model obtained with RNAs is important. This correlates well all thermodynamic variables with the saturation temperature. The aim of this study was to model the thermodynamic properties: saturation pressure, enthalpy, internal energy and entropy of liquid and saturated steam through RNAs. For the development of RNAs a Matlab software 7.5 was used. The architecture used was MPL ( multilayer perceptron). The transfer function used in hidden neurons was the sigmoid and the output was a linear function. The Levenberg-Marquardt backpropagation algorithm was used for the training and learning process of the network. The best fit of the data $(R=0,999999$, MSE $=4,57)$ was obtained with the use of 7 hidden neurons in the model, which allows us to obtain the thermodynamic properties of liquid and saturated water steam just by entering the saturation temperature. A mathematical model of neural network was found, which competes with traditional thermodynamic models.

Keywords: neural network, saturate steam, thermodynamic property, mathematical model.

\section{INTRODUCCIÓN}

Las redes neuronales artificiales son estructuras computacionales inspiradas en los sistemas neuronales biológicos. Los modelos computacionales convencionales son particularmente bien utilizados para ejecutar secuencias de instrucciones que han sido precisamente formuladas para ello. Por otro lado, los sistemas neuronales biológicos son bien utilizados para tareas/operaciones tales como el habla, la visión, generalización y reconocimiento de patrones espaciales y temporales complejos en presencia de datos con ruidos, los cuales son extremadamente difíciles de conseguir con los métodos computacionales convencionales. Por lo tanto, la motivación por las redes neuronales artificiales es lograr muchas de estas habilidades de los sistemas neuronales biológicos. Una RNA es un enorme procesador distribuido en paralelo hecho de unas unidades de proceso simples, las cuales tienen una fuerte tendencia natural por el conocimiento empírico, las RNAs tienen la habilidad de aprender por ejemplos y no requieren un conocimiento previo de la relación de los parámetros o variables, además son capaces de trabajar con incertidumbres, ruido $y$ relaciones no lineales. Cada vez más, las
RNAs están siendo usadas como herramientas para realizar regresiones no lineales y para desarrollar modelos con relaciones de las variables muy complejas. Una de las ventajas de las RNAs con respecto a los métodos estadísticos convencionales es que no requieren suposiciones sobre la distribución de los datos a analizar (Ponce 2010, Farid 2010). Existen diferentes problemas en los procesos de alimentos y bioprocesos que no pueden ser caracterizados ni resueltos usando aproximaciones con modelos de base física. En estas situaciones el modelamiento con RNAs pueden ser usadas como alternativas potenciales (Farid 2010). Mientras el uso de los modelos fenomenológicos requiere una solución simultánea de un gran número de ecuaciones algebraicas no lineales que necesitan procesos iterativos largos $y$ exhaustivos, la solución basada en técnicas de redes neuronales artificiales es más simple y rápida, y consiste en una solución de un sistema de ecuaciones algebraicas lineales. Esta característica hace a las redes neuronales interesante en la aplicación de modelamiento, optimización y control de procesos complejos [12,1,5, 10]. Una gran ventaja de los modelos de redes neuronales es que no es necesario saber la relación entre las variables de entrada y de salida, éstas se encargan de buscar estas relaciones a través 
de entrenamientos sucesivos. Las redes neuronales son capaces de aprender con ejemplos, para incorporar muchas variables, y proveer rápida y apropiadamente respuestas a cualquier información nueva no presentada previamente [1].

Por otro lado, las propiedades termodinámicas son importantes en la modelación de los procesos, dado que con éstas se calculan los requerimientos de energía y la irreversibilidad que tiene dicho proceso. Las propiedades termodinámicas del líquido y vapor de agua saturados se pueden modelar a través de muchas ecuaciones, como la ecuación de Clapeyron, la ecuación de Clausius-Clapeyron, la ecuación de Antoinne, los modelos de Riedel y Watson entre otras, todas ellas arrojando valores cercanos o alejados del valor real y con cierto grado de correlación, además de esto cada ecuación arroja una sola propiedad termodinámica, razón por la cual el modelo obtenido con redes neuronales artificiales es importante, ya que éste correlaciona todas las variables termodinámicas mencionadas con la temperatura de saturación con un grado de ajuste bastante alto resultando un modelo que se puede utilizar en el diseño, control y modelamiento de procesos donde se utilice el vapor de agua como agente de calentamiento. El objetivo de este artículo fue modelar las propiedades termodinámicas presión de saturación, entalpía, energía interna y entropía del líquido y vapor de agua saturados por medio de redes neuronales artificiales.

\section{MATERIALES Y MÉTODOS}

Para el desarrollo de la red neuronal se utilizó Simulink de MatLab 7.5. (Figura 1), La capa de entrada de la red tuvo un (1) nodo correspondiente a la variable independiente: temperatura de saturación $(\mathrm{T})$. La capa de salida de la red tuvo siete (7) nodos correspondientes a las variables dependientes: presión de saturación $(P)$, energía interna $\left(U_{I}\right)$, entalpía $\left(\mathrm{HI}_{\mathrm{I}}\right.$ y entropía $(\mathrm{S} \mathrm{I})$ del líquido saturado y energía interna $\left(U_{v}\right)$, entalpía $\left(H_{v}\right)$ y entropía $\left(S_{v}\right)$ del vapor saturado.

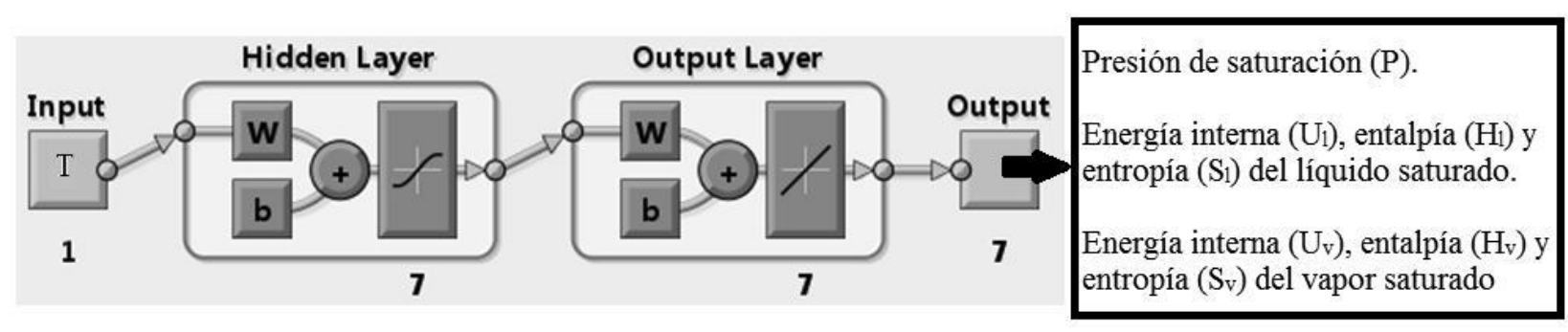

Figura 1. Diagrama de la Red Neuronal en Matlab.

La función de transferencia de la arquitectura MLP (perceptrón multicapa) usada en las neuronas ocultas fue la sigmoidea y en las de salida fue lineal. El algoritmo LevenbergMarquardt backpropagation fue usado para el entrenamiento y proceso de aprendizaje de la red ejecutándose en 437 iteraciones. La mejor topología, la velocidad de aprendizaje y el número de capas ocultas con su respectivo número de neuronas fueron determinados por la técnica de ensayo y error que resultó en la validación del error mínimo. Para el entrenamiento de la red fue usado un set de 173 datos en el rango de 30 a $370{ }^{\circ} \mathrm{C}$ obtenidos de las tablas de vapor de agua saturada de Smith et al. (2007). Simultáneamente, fueron usados 74 datos para la validación y prueba de la red.

\section{RESULTADOS Y DISCUSIÓN}

El mejor ajuste de los datos durante el entrenamiento, validación y test de la red neuronal $\left(R^{2}=0,999999, M S E=4,57\right)$ (Figura2), se obtuvo con la utilización de 7 neuronas ocultas. 
Fabián Ortega, Redes Neuronales Artificiales y predicción de propiedades

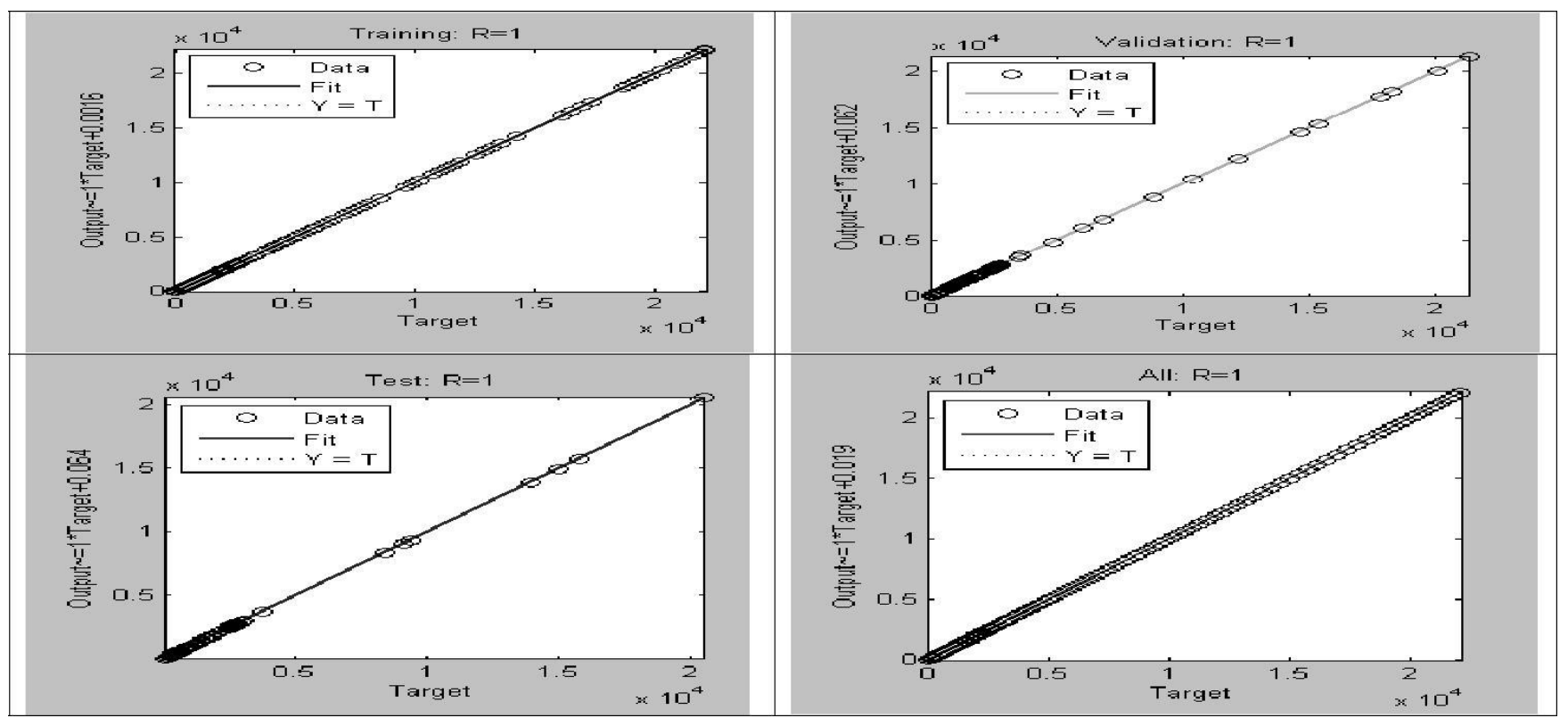

Figura 2. Ajuste de entrenamiento, validación, test y total de la red neuronal.

Modelo de red neuronal obtenido se puede usar en el rango evaluado con un error medio relativo menor al $2,2 \%$ en cada una de las propiedades evaluadas. Los errores de los calores latentes de vaporización calculados con el modelo de RNA (tabla1), obtenido no superan el $0,35 \%$ mientras que Smith et al. (2007) reportan que la ecuación de Riedel presenta error del 3,4\% y la ecuación de Watson presenta errores que en algunos casos superan el $5 \%$. Por otro lado, la ecuación de Clausius-Clapeyron para el cálculo del calor latente de vaporización está restringida para presiones bajas lejos del punto crítico y los errores obtenidos pueden superar el $5 \%$, mientras que el modelo de RNA obtenido se puede utilizar a presiones cercanas al punto crítico del agua, demostrándose con esto el gran ajuste de las RNAs con los datos reales.

Tabla 1. Errores medios relativos de la red neuronal.

\begin{tabular}{|lc|}
\hline \multicolumn{1}{|c|}{ Variable termodinámica } & \% Error medio relativo \\
\hline Presión de saturación & 2,12 \\
Energía interna del líquido saturado & 0,17 \\
Energía interna del vapor saturado & 0,03 \\
Entalpía del líquido saturado & 0,17 \\
Entalpía del vapor saturado & 0,03 \\
Entropía del líquido saturado & 0,68 \\
Entropía del vapor saturado & 2,15 \\
\hline
\end{tabular}

El esquema elaborado en Simulink (Figura3), junto con los coeficientes matriciales de pesos y constantes es mostrado a continuación: 


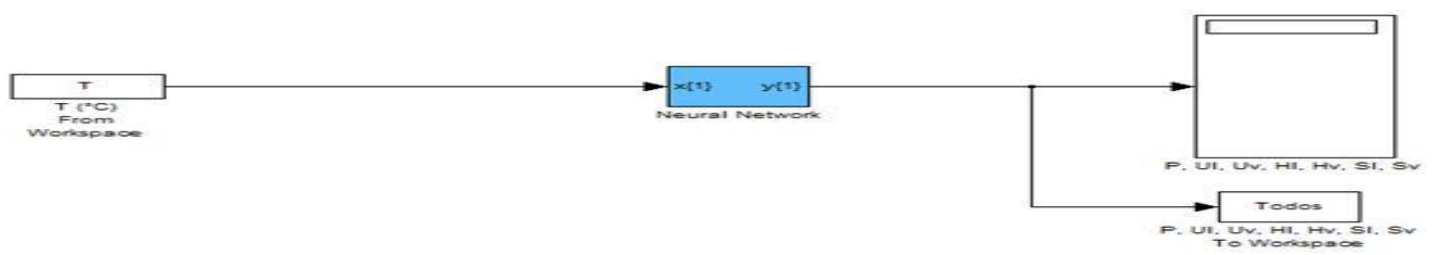

Figura 3. Modelo general en Simulink

Entrada y salidas:

Entrada $=\mathrm{T}\left({ }^{\circ} \mathrm{C}\right)$

Orden de salidas $=\mathrm{P}(\mathrm{kPa}), \mathrm{UI}\left(\mathrm{KJ} \mathrm{Kg}^{-1}\right)$, Uv $\left(\mathrm{KJ} \mathrm{Kg}^{-1}\right), \mathrm{HI}\left(\mathrm{KJ} \mathrm{Kg}^{-1}\right), \mathrm{Hv}\left(\mathrm{KJ} \mathrm{Kg}^{-1}\right)$, $\mathrm{SI}\left(\mathrm{KJ} \mathrm{Kg}^{-1} \mathrm{~K}\right)$, Sv $\left(\mathrm{KJ} \mathrm{Kg}^{-1} \mathrm{~K}\right)$

Esquema general dentro de la red en Simulink (Neural Network) (Figura 4):
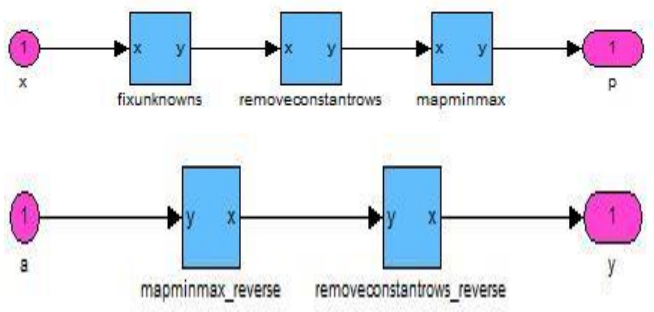

PROCESS OUTPUT 1:

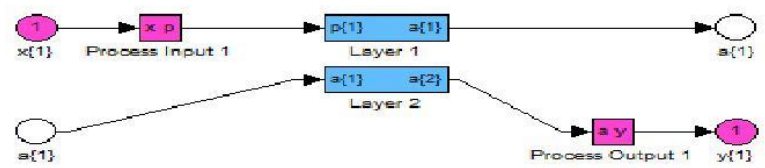

Figura 4. Neural Network

PROCESS INPUT 1:
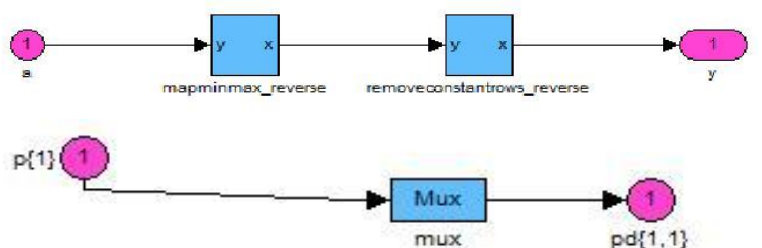

\section{LAYER1:}

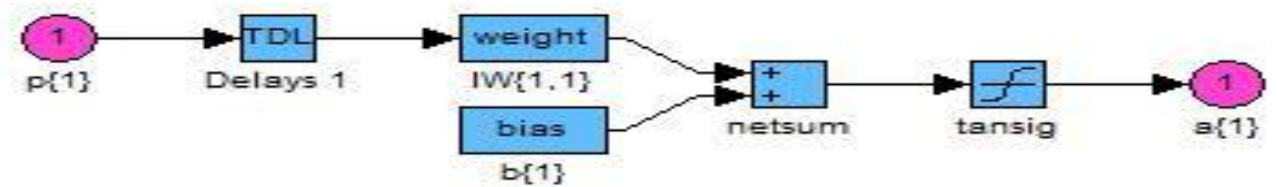

Figura 5: simulink General

Con los resultados obtenidos se muestra que la arquitectura MLP con una única capa oculta por cada variable dependiente es un aproximador con un alto grado de ajuste de las funciones termodinámicas propuestas, corroborando lo expresado por Hecht-Nielsen [4].

\section{CONCLUSIÓN}

Se obtuvo un modelo matemático de red neuronal en Simulink (MatLab) con un alto grado de ajuste y que compite con los modelos termodinámicos tradicionales, el cual permite obtener las propiedades termodinámicas ( $P$, $\mathrm{U}_{\mathrm{l}}, \mathrm{U}_{\mathrm{v}}, \mathrm{H}_{\mathrm{l}}, \mathrm{H}_{\mathrm{v}}, \mathrm{S}, \mathrm{S}_{\mathrm{v}}$ ) del líquido y vapor de agua saturados con sólo entrar la temperatura de saturación.

\section{REFERENCIAS}

[1]. Adhikari, B. y Jindal, V. K. 2000. Artificial neural networks: a new tool for prediction of pressure drop of non-Newtonian fluid foods through tubes. Journal of Food Engineering 46: 43-51. 
[2]. Farid, M. 2010. Mathematical Modeling of Food Processing. Editorial CRC Press, USA, p901-921.

[3]. García, P. 2009. Introducción a las redes neuronales y su aplicación a la investigación en Astrofísica. Editorial Universidad de Gran Canarias, España, p45-57

[4]. Hilera, J.R. and Martínez, V.J. 1995. Redes neuronales artificiales: fundamentos, modelos y aplicaciones. Ediciones Rama, España, p76-98.

[5]. Isazi, P. 2002. Redes neuronales Artificiales. Primera edición Ed. Prentice Hall Latinoamérica, México, p100-112.

[6]. Mittal, G. S. and Zhang, J. 2000.

Prediction of freezing time for food products using a neural network, Food Research International 33: 557-562.

[7]. Mittal, G. S. and Zhang, J. 2001. Artificial neural network for the prediction of temperature, moisture and fat contents in meatballs during deep-fat frying, International Journal of Food Science and Technology 36: 489-497.

[8]. Ponce, P. 2010. Inteligencia artificial con aplicaciones a la Ingeniería. Editorial Alfaomega, México, p6-12.

[9]. Smith, J.M., Van Ness, H.C. and Abbott, M.M. 2007. Introducción a la termodinámica en Ingeniería Química. Séptima edición. Mc Graw Hill Interamericana Editores, México, p635662.

[10]. Zbicinski, I. and Ciesielski, K. 2002. Extension of the neural networks operating range by the application of dimensionless numbers in prediction of heat transfer coefficient, Drying Technology 